\title{
Fixed-dose combination antituberculosis therapy: a systematic review and meta-analysis
}

\author{
Amr S. Albanna1, Benjamin M. Smith¹, Deanna Cowan² and Dick Menzies \\ Affiliations: \\ 'Respiratory Epidemiology and Clinical Research Unit, Montreal Chest Institute, McGill University, Montreal, QC, and \\ ${ }^{2}$ Life Sciences Library, McGill University, Montreal, QC, Canada.

\section{Correspondence:} \\ D. Menzies, Room K1.24, Montreal Chest Institute, 3650 St Urbain St, Montreal, QC, H2X 2P4, Canada. \\ E-mail: dick.menziesamcgill.ca
}

ABSTRACT Fixed-dose combination (FDC) formulations are currently recommended for the treatment of active tuberculosis (TB). We have conducted a systematic review to evaluate the risk of treatment failure or disease relapse, acquired drug resistance, bacterial conversion after 2 months of treatment, adverse events, adherence and treatment satisfaction associated with treatment of active TB using FDC or separate drug formulations.

We searched four electronic databases for randomised controlled trials and cohort studies. Results from trials that directly compared FDC to separate drug formulations were pooled. Results from other studies were reported separately.

We identified 2450 citations from which 15 controlled trials and four additional relevant studies were included. In the 15 trials there were no differences in acquired drug resistance, bacterial conversion after 2 months of treatment or adverse drug reactions with FDC or separate drug formulations. There was a trend toward higher risk of failure or relapse with FDC (pooled relative risk 1.28 (95\% CI 0.99-1.7)). Based on individual study results, only one of two trials that assessed treatment satisfaction, and none of five that assessed patient adherence, favoured FDCs.

Although FDC formulations simplify TB therapy, the current evidence does not indicate that these formulations improve treatment outcomes among patients with active TB.

@ERSpublications

Current evidence does not indicate that fixed-dose combinations of first-line TB drugs improve treatment outcomes http://ow.ly/la48v

\footnotetext{
This article has supplementary material available from www.erj.ersjournals.com

Received: Nov 082012 | Accepted after revision: Dec 202012 | First published online: Jan 112013

Support statement: A.S. Albanna received salary support from the Ministry of Higher Education, Government of Saudi Arabia.

Conflict of interest: None declared.

Copyright @ERS 2013
} 


\section{Introduction}

Tuberculosis (TB) is a global health problem, with 8.7 million new cases and accounting for $\sim 1.4$ million deaths annually [1]. Moreover, strains of Mycobacterium tuberculosis that are resistant to standard anti-TB therapy are emerging in almost all areas reporting to the World Health Organization (WHO) [2]. Nonadherence to treatment regimen and inappropriate prescription of TB therapy are believed to be major contributing factors to this public health problem [3, 4]. Due to the large number of tablets used in the treatment regimens of TB, fixed-dose combination (FDC) tablets, each combining two or more anti-TB drugs, have been manufactured since the 1980s [5] to simplify TB therapy and facilitate physician and patient compliance with treatment recommendations [6]. These FDC tablets also prevent inadvertent monotherapy, which may occur because of physician error in prescription, inadequate regimens or patient error in selectively taking only one drug. In addition, dealing with one combined formulation that contains all essential drugs simplifies drug procurement, storage and distribution, and may consequently reduce drug supply management errors and cost.

In 1994, the WHO and the International Union against Tuberculosis and Lung Disease (IUATLD) recommended the use of FDC anti-TB therapy [7]. Following the announcement of this recommendation, and its more widespread implementation, concerns were raised about adequate bioavailability of the component drugs, particularly rifampicin (RIF) due to its enhanced decomposition in the presence of isoniazid (INH) [8-11]. As a result, the WHO and the IUATLD established guidelines for assuring the bioavailability of FDC anti-TB drug components [12]. Currently, the WHO Model List of Essential Drugs includes two-drug formulations (INH + RIF and INH + ethambutol), three-drug formulations (INH + RIF + ethambutol and INH + RIF + pyrazinamide) and a four-drug formulation (INH + RIF + ethambutol + pyrazinamide) [13].

Despite the anticipated advantages of FDC anti-TB drugs, questions about their effectiveness have not been answered. Many observational studies and clinical trials have been conducted to assess the effectiveness of FDC drugs in reducing treatment failure, disease relapse and the emergence of drug resistance. Among these studies, the use of FDC drugs has resulted in favourable [14], unfavourable [15] or unchanged treatment outcomes $[16,17]$.

Due to the anticipated advantages, and despite the current conflicting evidence, the FDC formulations are recommended for treatment of active TB by the WHO [18], the International Standards for TB Care (Standard 8) [19], and the American Thoracic Society [20].

\section{Study questions}

The study aimed to answer the following: 1) in patients who are treated for bacteriologically confirmed TB, is anti-TB therapy using FDC drug formulations associated with lower rates of bacteriologically confirmed treatment failure, disease relapse or emergence of drug resistance when compared to separate-drug formulations?; and 2) in patients receiving TB treatment, are adverse drug reactions, patient adherence and treatment satisfaction superior with FDC than separate-drug formulations?

\section{Methods}

\section{Search strategy and study selection}

A search strategy was designed to retrieve articles investigating FDC anti-TB therapy published in any language between January 1980 and July 2011. The databases used for the literature search were MEDLINE (Ovid platform); MEDLINE In-Process \& Other Non-Indexed Citations (Ovid platform); Embase (Ovid platform); The Cochrane Library (published by Wiley), which includes Cochrane Reviews, DARE and Central Register of Controlled Clinical Trials; and LILACS (BIREME, PAHO and WHO Latin-American and Caribbean Center on Health Sciences Information) databases. The following four sets of search terms were combined with "AND": 1) terms about TB, Mycobacterium and anti-TB; 2) terms to restrict for treatment regimens that contain both isoniazid and rifampicin; 3) terms to restrict for the use of combination formulations; and 4) restriction to human studies published since 1980. For more details about the terms used in each database, refer to the online supplementary material.

Studies that fulfilled all of the following criteria were eligible for full-text review: 1) randomised clinical trial (RCT) or cohort study (the latter should include $\geqslant 50$ subjects); 2) bacteriologically confirmed diagnosis of active TB, based on culture or smear analyses, among included subjects; 3 ) treatment with an FDC anti-TB formulation that contained at least RIF and INH; 4) treatment with an effective anti-TB regimen (i.e. daily or at least three times weekly administration of RIF and INH for 9 months, or for 6 months when pyrazinamide was added during the initial 2 months); 5) measurement of at least one of our primary treatment outcomes (i.e. bacteriologically confirmed treatment failure or relapse, or acquired drug 
resistance with diagnosis based on baseline and follow-up drug sensitivity testing); and 6) follow-up period of $\geqslant 5$ months during the treatment.

Selection of eligible studies was performed in a stepwise fashion: titles, then abstracts, then full texts, by two reviewers (A. Albanna and B. Smith) working independently. At each stage, all studies selected by either reviewer (i.e. concordant eligible or discordant) were included for full-text review. Inclusion of studies, after full-text review, was based on concordance of the two reviewers; disagreement was resolved by a third reviewer (D. Menzies).

\section{Data extraction}

The extracted data included information about the context of the study (study design, location and time period), characteristics of included subjects (age, sex, past TB treatment, HIV status and comorbidities), disease status (disease site and drug sensitivity) and treatment outcomes (completion of treatment, compliance to treatment, adverse drug reaction, treatment failure, death during treatment, disease relapse, acquired drug resistance and patient satisfaction). In addition, a quality assessment scale was adapted from the Cochrane Collaboration tool to assess the following five quality indicators: 1) sequential or randomised allocation of subjects to study groups; 2) concealment of the allocation, in case of RCTs; 3) adequate assessment of incomplete outcome data; 4) reporting of pre-specified or all expected outcomes (to obviate the possibility of selective outcome reporting); and 5) adequate consideration of potential sources of bias. To ensure accurate and consistent data collection, both reviewers independently performed data extraction from a sample of nine articles. Important missing data were obtained by correspondence with the studies' authors through email contact.

\section{Outcome measures}

The pre-specified primary outcome measures were "treatment failure or disease relapse", as one outcome, and acquired drug resistance as another. The pre-specified secondary outcomes were bacterial conversion after 2 months of treatment, adverse drug reaction, patient adherence and treatment satisfaction. Prespecified subgroup analysis was stratified by baseline drug sensitivity testing, study quality, publication year, treatment supervision modality, type of treatment regimen and FDC formulation/producer. Our decision to stratify the studies by their potential conflict of interest was made after collecting the data (post hoc analysis).

\section{Data analysis}

Differences in the outcomes between the comparative groups were expressed as risk ratios and 95\% confidence intervals, using per-protocol analysis. The effect measures of comparative RCTs were pooled using the DerSimonian-Laird random effects model. The use of a random effects, rather than a fixed effect, model was pre-specified to account for variations between studies related to the type and severity of prevalent disease, standard of care and research quality. To obtain valid, unbiased comparative estimates, our analysis focused on the comparative RCTs, which represented the majority of the included studies. Summaries of the effect measures from the other studies were not pooled and were reported separately. Between-study heterogeneity was assessed using Chi-squared (Cochran's Q), indicating statistical significance as $\mathrm{p}<0.1$ and I-squared tests. The latter are interpreted as showing unimportant heterogeneity if values are $<40 \%$, moderate heterogeneity if values are between $40 \%$ and $60 \%$ and substantial heterogeneity if values $>60 \%$. In the case of moderate or substantial heterogeneity of results, or inconsistent methods of ascertainment across studies, the outcome estimates were not pooled and were reported separately. Subgroup and meta-regression analyses were performed to detect factors that influenced the primary outcome results. Reporting bias, which includes publication bias, was assessed using funnel plot and Egger's test, which is based on linear regression analysis to test the association between the intervention effect (using logarithmic scale) and its standard error [21]. All analyses were conducted using STATA (version 12) (StataCorp, College Station, TX, USA) software.

\section{Results}

Of 2450 citations identified by our search strategy, 25 met the inclusion criteria for this review. These 25 articles reported results of 19 different studies (fig. 1). Among these 19 studies, 15 RCTs directly compared FDC to separate drug formulations and included a total of 5630 subjects (table 1). The other four studies represent one comparative cohort [39], two noncomparative (i.e. no direct comparison between FDC and separate drug formulations) RCTs [40-42] and one noncomparative cohort [43] which included total numbers of 474, 310 and 1888 subjects, respectively; refer to the online supplementary material for study descriptions. 


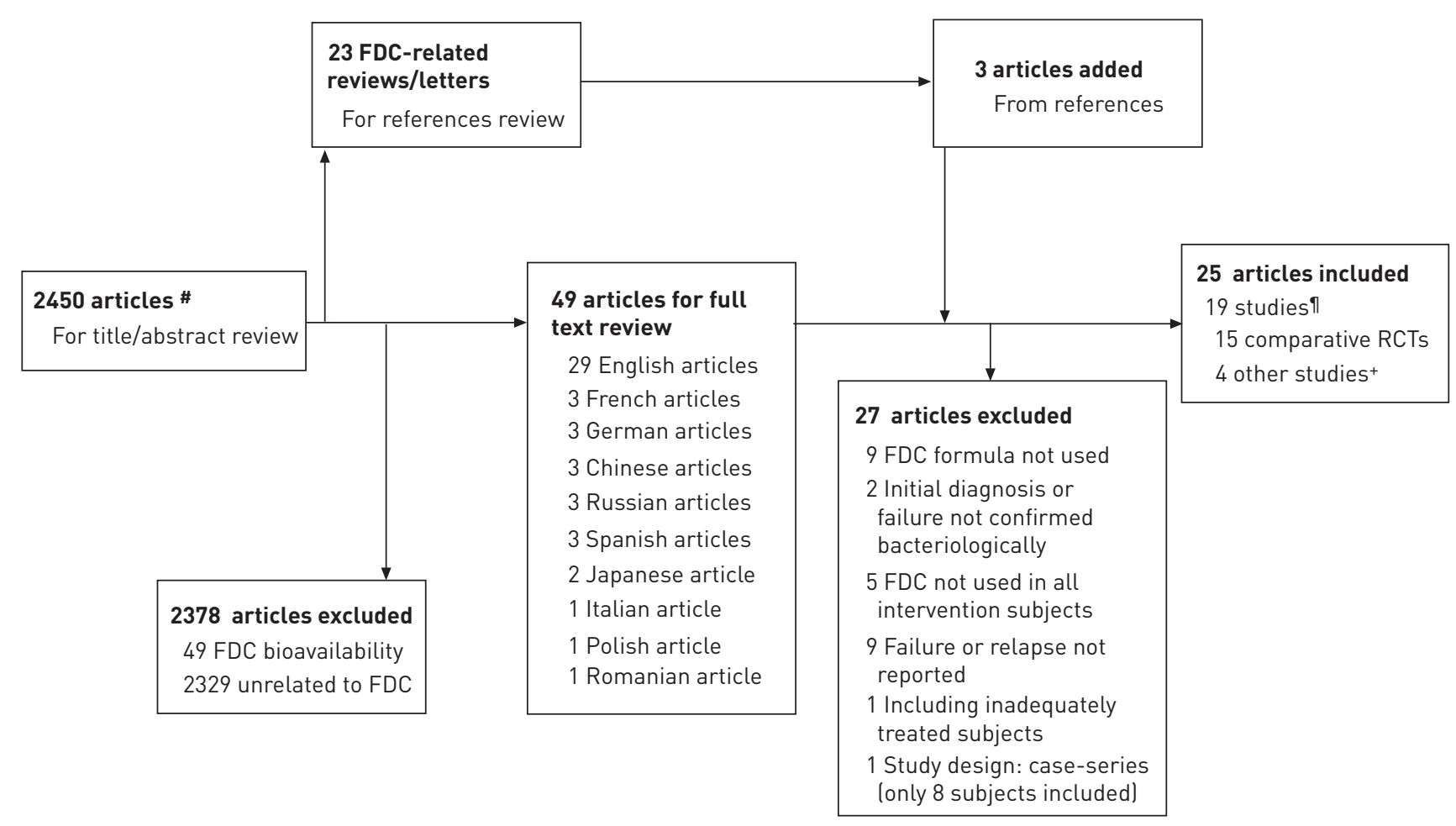

FIGURE 1 Study selection. FDC: fixed-dose combination; RCTs: randomised controlled trials. ${ }^{\#}$ : After excluding duplicate articles; ${ }^{\circ}$ : some studies were published in more than one article; ${ }^{+}$: one comparative cohort and three noncomparative studies.

Primary outcome results of the comparative RCTs

In the 15 RCTs there was a trend toward higher risk of treatment failure or disease relapse with FDC compared to separate drug formulations (pooled relative risk 1.28 (95\% CI 0.99-1.7)), with no significant heterogeneity between the results of different studies (fig. 2). The incidence of failure or relapse was relatively low in both treatment arms (table 2), and the pooled risk difference was $1 \%$ (95\% CI $-0.2-2 \%$ ) higher with FDCs.

As seen in table 2, the risk of acquired drug resistance, based on pooled results from four RCTs, was very low in both treatment arms and the relative risk estimate was inconclusive.

In the subgroup analyses, baseline drug sensitivity status appeared to modify the risk of "treatment failure or disease relapse". Comparing FDC with separate-drug formulations, the risk was significantly higher with FDCs within the stratum of subjects with baseline drug-susceptible TB (pooled risk ratio 1.48 (95\% CI 1.04-2)), and lower, although not significantly so, with FDCs within the drug-resistant stratum. In addition, FDC formulation was inferior to separate-drug formulation among subjects receiving self-administered therapy and in studies with no potential conflict of interest (fig. 3).

Univariate metaregression analyses did not indicate a significant influence of publication year or study quality on the outcome results (fig. 4). After including these two covariates with drug susceptibility, treatment supervision and potential conflict of interest variables in a multivariate metaregression model, drug susceptibility was the only variable that significantly modified the outcome results (comparing the point estimate within drug-resistant to the point estimate within drug-susceptible strata, risk ratio 0.32 (95\% CI 0.11-0.94); $\mathrm{p}=0.04)$.

Funnel plot analysis demonstrated a symmetrical distribution of "treatment failure or relapse" effect estimates across studies and the regression line indicated that small studies, which have less precise estimates (larger standard errors), tended to shift the treatment effect in favour of FDC treatment (fig, 5). However, the small-study effect was not significant (estimated bias coefficient -0.36 (95\% CI -1.2-0.49); $\mathrm{p}=0.39$ ).

\section{Secondary outcome results of comparative RCTs}

As seen in table 2, FDC treatment was almost similar to separate-formulation treatment for eliminating mycobacterial isolation after 2 months of treatment and had similar association with adverse drug reaction. The estimated results of patient adherence and treatment satisfaction outcomes were not pooled because of 


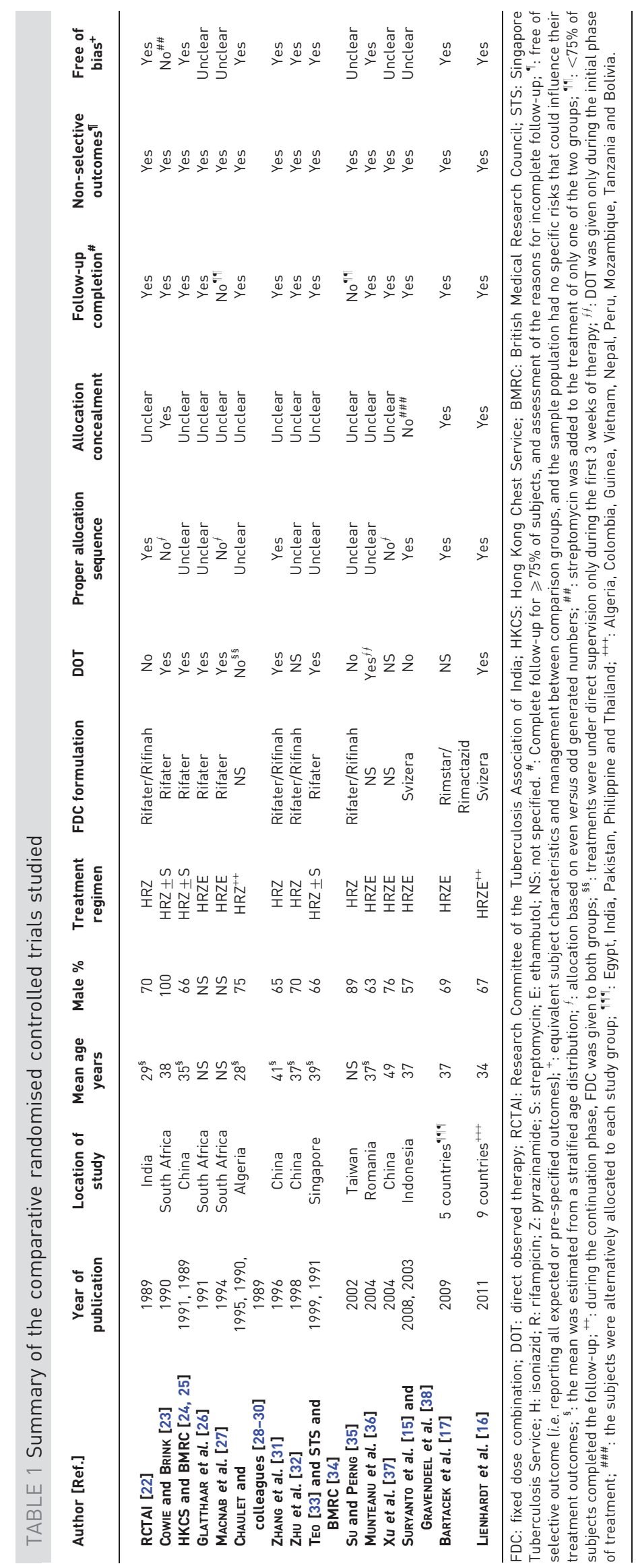




\section{Drug sensitive}

RCTAI [22]

CoWIE and BRINK [23]

HKCS and BMRC [24]

Chaulet et al. [28]

TEO [33]

LIENHARDT et al. [16]

MACNAB et al. [27]

ZHu et al. [32]

Munteanu et al. [36]

Subtotal (I-squared $=0.0 \%, p=0.661$ )

\section{Drug resistant}

RCTAI [22]

HKCS and BMRC [24]

Chaulet et al. [28]

ZHu et al. [32]

TEO [33]

LIENHARDT et al. [16]

Subtotal (I-squared $=0.0 \%, p=0.690$ )

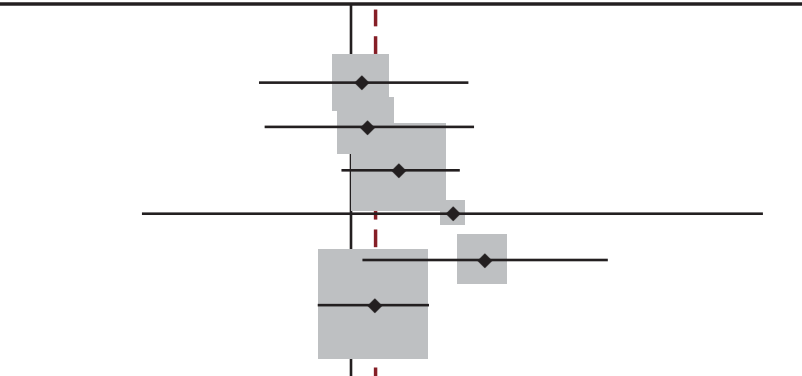

$1.12(0.38-3.31) \quad 5.56$

$1.18(0.40-3.47) \quad 5.58$

$1.64(0.88-3.06) \quad 16.71$

$2.82(0.12-68.18) \quad 0.64$

$3.86(1.10-13.53) \quad 4.14$

$1.26(0.72-2.21) \quad 20.96$

Excluded $\quad 0.00$

Excluded $\quad 0.00$

Excluded $\quad 0.00$

$1.48(1.04-2.09) \quad 53.58$

$1.64(0.18-15.26)$

$0.24(0.03-1.79) \quad 1.61$

$2.00(0.18-22.06) \quad 1.13$

$0.34(0.07-1.73) \quad 2.49$

$0.70(0.05-9.41) \quad 0.96$

$0.64(0.19-2.15) \quad 4.40$

$0.61(0.29-1.28) \quad 11.89$

\section{Not stratified}

GLATTHAAR et al. [26]

ZHANG et al. [31]

Su and Perng [35]

$\mathrm{Xu}$ et al. [37]

SURYANTO et al. [15]

BARTACEK et al. [17]

Subtotal (I-squared $=18.0 \%, p=0.297)$

Overall (I-squared $=0.0 \%, p=0.464)$

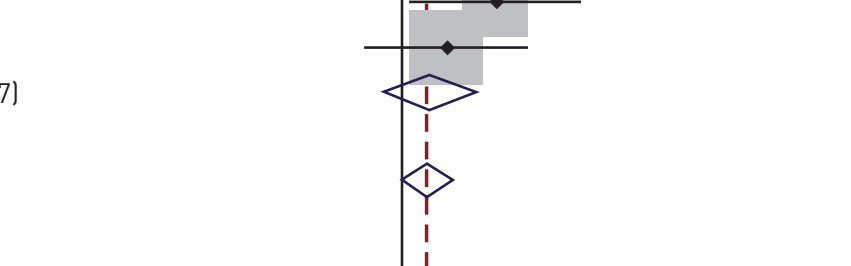

$0.68(0.30-1.57) \quad 9.33$

$0.76(0.17-3.30) \quad 3.00$

$4.81(0.24-95.58) \quad 0.73$

$1.15(0.31-4.20) \quad 3.85$

$2.58(1.05-6.33) \quad 8.09$

$1.59(0.70-3.64) \quad 9.53$

$1.31(0.80-2.16) \quad 34.52$

$1.28(0.99-1.65) \quad 100.00$

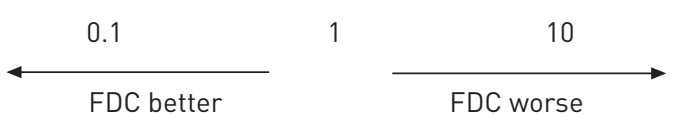

FIGURE 2 Forest plot of risk ratios of failure or relapse (main outcome) among fixed-dose combination (FDC) versus separate drug formulation groups, stratified by baseline drug susceptibility testing. RCTAI: Research Committee of the Tuberculosis Association of India; HKCS: Hong Kong Chest Service; BMRC: British Medical Research Council. ": From random effects analysis. ": Zero events in both arms, hence risk ratio was not estimated. When including these studies and adding 0.5 to each cell of the $2 \times 2$ table, the pooled risk ratio of the randomised controlled trials within the drug-sensitive stratum was 1.45 ( $95 \%$ CI $1.03-2.04$ ), and the overall risk ratio was 1.26 (95\% CI $0.98-1.63)$.

inconsistent ascertainment methods and significant heterogeneity of results (I-squared $67 \%$ and $98 \%$, respectively) across the included RCTs. Only one of two RCTs that assessed treatment satisfaction, and none of five that assessed patient adherence, favoured FDCs.

\section{Outcome results of the cohort and noncomparative studies}

Among included studies, the comparative cohort [39] presented the highest proportion of "treatment failure or disease relapse" outcome, ranging from 5\% to $11 \%$ among drug-susceptible and from $21 \%$ to $35 \%$ among drug-resistant TB patients. The crude risk ratio comparing FDC to separate-formulation treatments was 0.46 (95\% CI 0.2-0.98) among drug-susceptible and 0.6 (95\% CI 0.2-1.5) among drugresistant TB patients. Results from the noncomparative studies [40-43] indicated a low proportion of 


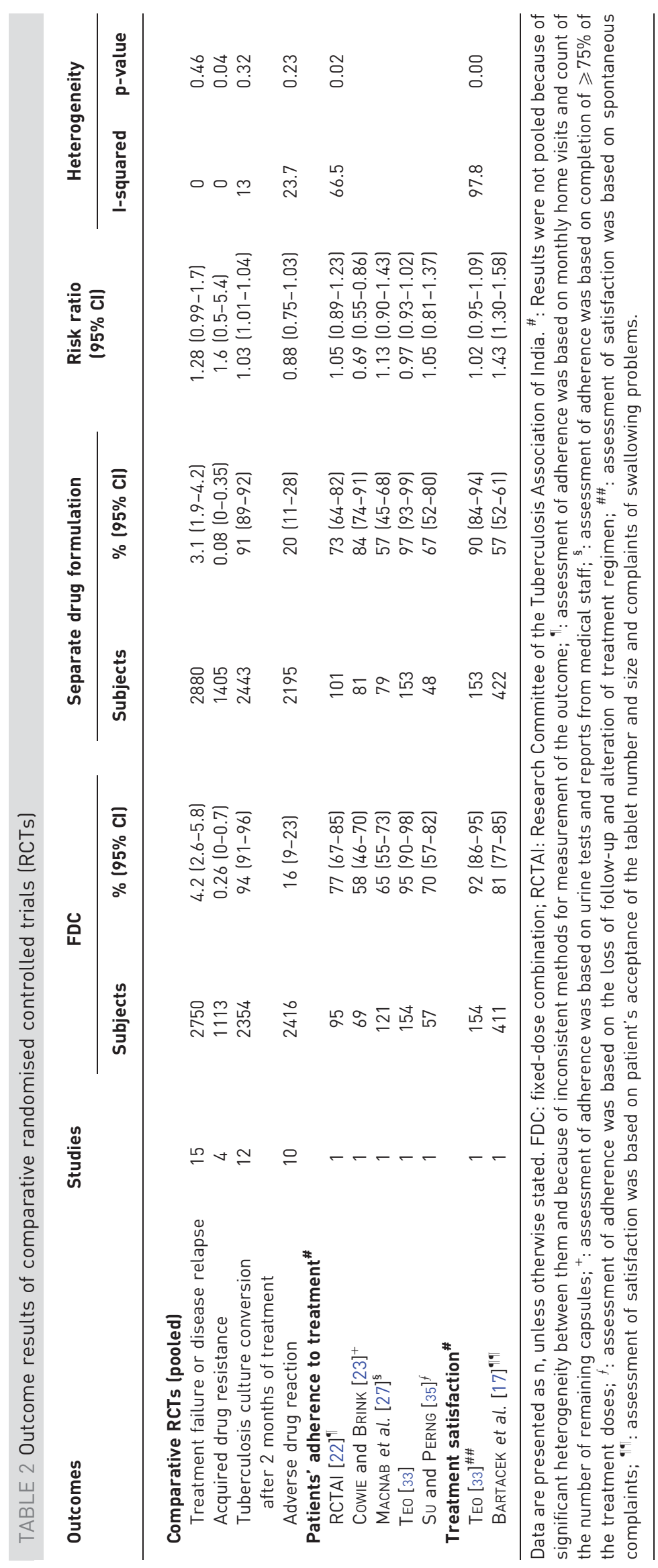




\section{Treatment regimen}

$\begin{array}{lll}H R Z & 4 & 0.95(0.48-1.87) \\ H R Z \pm S & 3 & 1.42(0.72-2.79) \\ H R Z E & 6 & 1.45(0.97-2.19) \\ H R Z \pm E & 2 & 0.79(0.39-1.60)\end{array}$

\section{FDC formulation/ producer}

$\begin{array}{lll}\text { Rifater } & 9 & 1.11(0.73-1.70) \\ \text { Rimstar } & 1 & 1.59(0.70-3.64) \\ \text { Svizera } & 2 & 1.37(0.71-2.62) \\ \text { NS } & 3 & 1.42(0.48-4.17)\end{array}$

\section{Supervision of TB treatment}

$\begin{array}{lcc}\text { Directly observed } & 8 & 1.13(0.78-1.65) \\ \text { Self-administered } & 4 & \mathbf{1 . 9 4}(\mathbf{1 . 0 5 - 3 . 5 7 )} \\ \text { NS } & 3 & 1.06(0.47-2.37)\end{array}$

\section{Drug susceptibility results}

Drug sensitive

Drug resistant

Mixed/unknown
Potential conflict of interest \#

Yes
No
Unclear

$1.48(1.04-2.09)$

$0.61(0.29-1.28)$

$1.31(0.80-2.16)$

$1.09(0.74-1.61)$

$1.56(1.00-2.42)$

$1.18(0.56-2.49)$

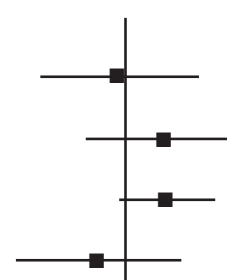

$\begin{array}{ll}5 & 1.09(0.74-1.61) \\ 4 & 1.56(1.00-2.42) \\ 6 & 1.18(0.56-2.49)\end{array}$

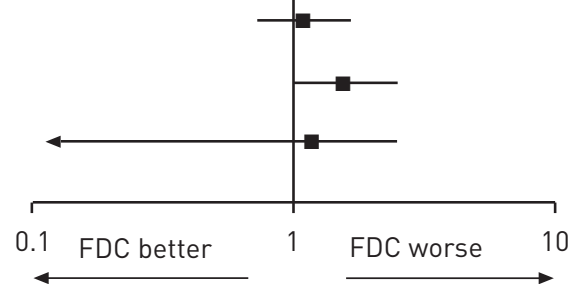

FIGURE 3 Subgroup analysis of the risk ratio of "treatment failure or disease relapse" among patients treated with fixeddose combinations (FDC) or separate drug formulations. Bold type represents statistical significance. H: isoniazid; R: rifampicin; Z: pyrazinamide; S: streptomycin; E: ethambutol; TB: tuberculosis; NS: not specified. \#: including funds and/or drug supplies.

"treatment failure or disease relapse", ranging from $0.5 \%$ to $2 \%$, and acquired drug resistance, ranging from 0 to $0.3 \%$, among TB treated patients; for details refer to the online supplementary materials.

\section{Discussion}

Based on pooled results of RCTs, FDC therapy was associated with a trend toward increased risk of treatment failure or disease relapse, statistically insignificant difference in the emergence of drug resistance and adverse drug reactions and clinically unimportant difference in culture conversion after 2 months of treatment. Although one study identified better treatment satisfaction, none of the included studies identified better patient adherence among TB patients treated with FDC compared to separate drug formulations.

While the pooled result of the RCTs suggests that FDC treatment does not reduce the risk of failure or relapse (risk ratio estimate with a lower 95\% CI range of 0.99 (close to the null value of 1.0)), it suggests potential increase in this risk (risk ratio estimate with an upper 95\% CI range of 1.7). This could be explained by reduced bioavailability of FDC component drugs [8-12], when compared to separate-drug formulations. Because these outcomes were infrequent, the absolute increased risk of failure or relapse with 
FIGURE 4 Univariate metaregression for estimating the effect of continuous covariates on the risk ratios of failure or relapse (main outcome) among fixeddose combination (FDC) versus separate drug formulation groups. a) Study publication year; b) study quality scale. The areas of the circles are inversely proportional to the variance. The study quality scale in figure b) ranges from 0 to 5 as the quality changes from low to high.
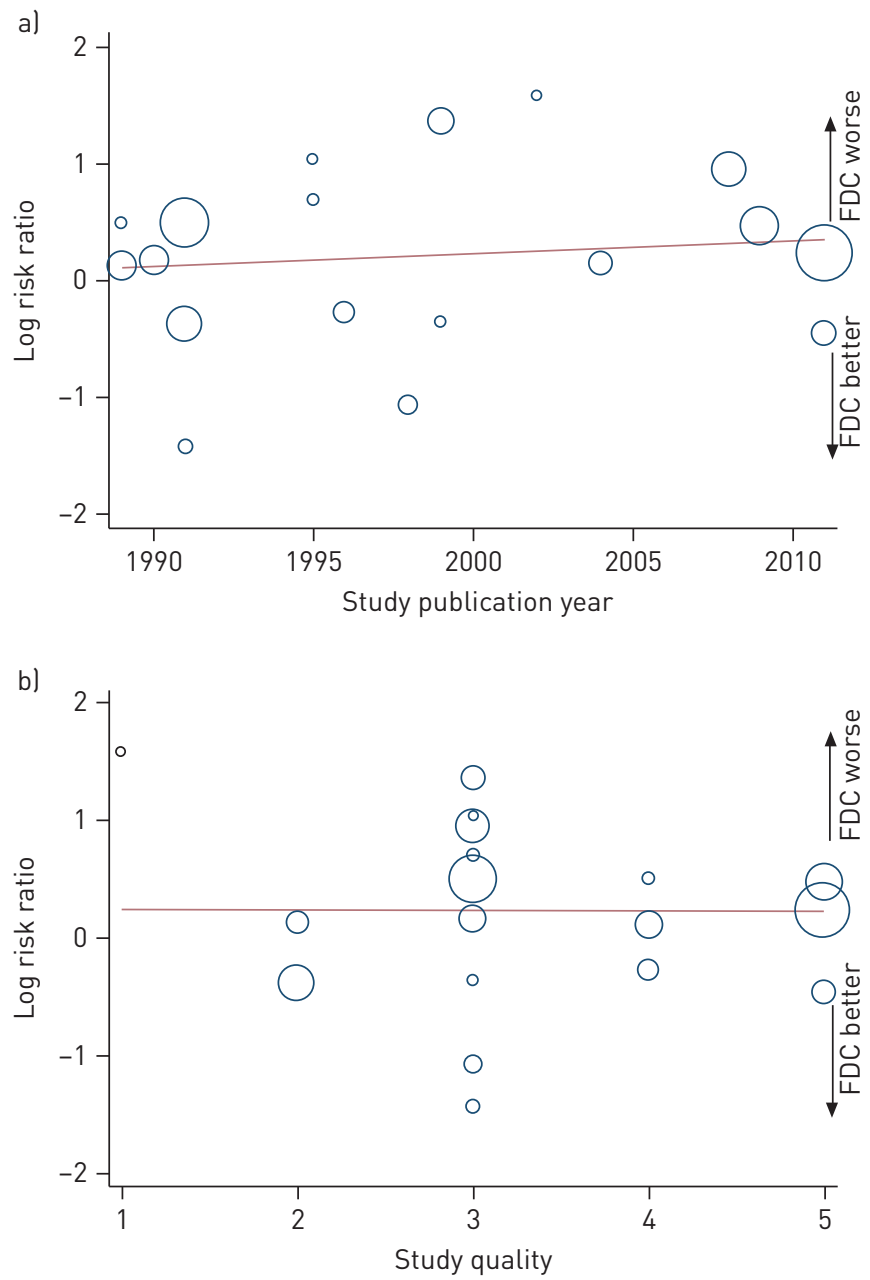

FDC treatment was only $1 \%$, with an upper $95 \%$ CI of $2 \%$. Using a noninferiority design, two of the included RCTs [16, 17] demonstrated a clinically insignificant risk of unfavourable outcomes with FDCs compared to separate-drug formulations. However, this study design does not address the question of whether or not FDCs improve treatment outcomes.

Despite the potential for providing the highest level of evidence in therapeutic intervention research, RCTs have been criticised because of limited generalisability of their results. RCTs are often conducted under optimal medical care and may underestimate the potential benefit of using FDC formulations to enhance adherence in settings where malpractice or unmonitored therapies are common. In spite of this limitation, however, important differences in adherence have been found in many randomised trials [44]. To better estimate treatment effectiveness, pragmatic clinical trials may be more appropriate as these trials are conducted in a way that more closely resembles usual clinical practice $[45,46]$.

We designed our research protocol to include observational studies, despite their inherent susceptibility to confounding, since they better reflect real medical practice. However, only one comparative cohort study [39], which presented crude estimates that were not adjusted for potential confounding, met the inclusion criteria. Failure to adjust for potential confounding in this observational study may have reduced the validity of results, since the use of FDC formulations may correlate with adherence to other standard treatment recommendations that influence disease outcomes. Because of this limitation and because the results of this comparative cohort were significantly different from the RCT results, we did not pool both results.

One of the limitations of this meta-analysis is the small number of studies that investigated the risk of acquired drug resistance, resulting in less precise estimates. Another limitation is the inconsistent ascertainment methods of patient adherence and treatment satisfaction in different studies; because of these heterogeneous methods, we did not pool these study results. In addition, we could not assess mortality as an 


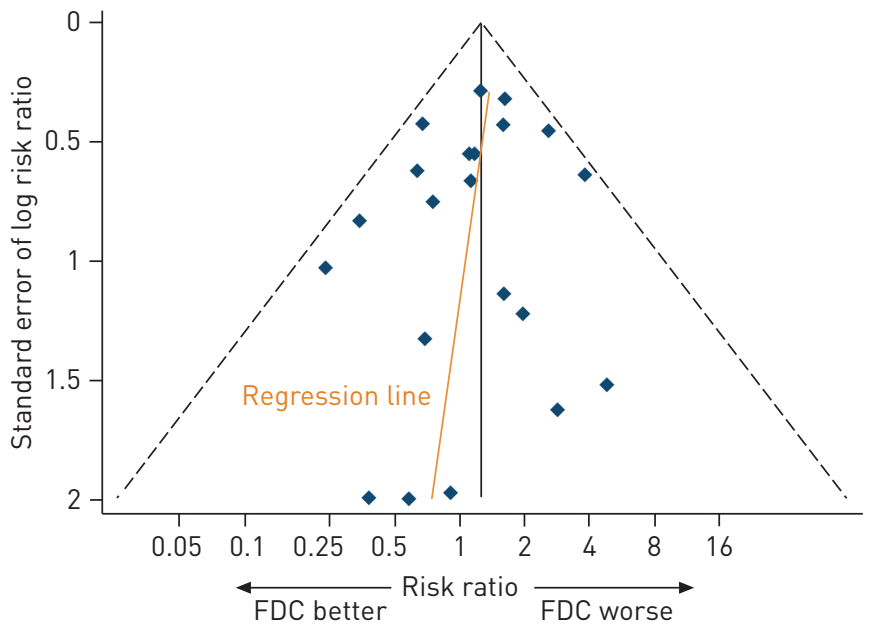

FIGURE 5 Funnel plot for the "treatment failure or disease relapse" outcome with pseudo 95\% confidence limits. FDC: fixed-dose combination. Egger's regression line represents the effect of smaller studies (higher standard error) as compared to the larger studies (lower standard error).

outcome because it was defined differently in the studies (all-cause versus TB-specific mortality), measured over different follow-up periods, ranging from 1 to 5 years, and in some studies was not reported or was not attributed to treatment group.

Despite these limitations, this systematic review has a number of strengths. Our systematic review was conducted without language restriction to accurately represent the existing evidence. Lack of significant heterogeneity of the estimates of treatment failure or disease relapse in the different trials permitted pooling and increased precision of our results. Another strength is the ability to stratify subjects based on their baseline drug susceptibility, which was a significant covariate factor influencing the risk of treatment failure or disease relapse. Comparing FDC to separate-drug formulation treatments, this risk tended to be higher within the stratum of subjects with baseline drug-susceptible TB and lower (in favour of FDC) within the stratum of subjects with baseline drug-resistant TB. This finding was unexpected because FDC formulations, which contain first-line anti-TB drugs, are inappropriate for patients with disease that is resistant to one or more of its component drugs. However, the result of the drug-resistant stratum included small numbers of patients with very heterogeneous forms of resistance to anti-TB drugs.

In summary, we used a strict search strategy to limit subjective selection of published studies; combined study results only when appropriate, using random effect meta-analysis which accounts for between-study variations; and followed the preferred reporting items for systematic reviews and meta-analyses (PRISMA) Statement [47] to report our data. Despite the advantage of FDC formulations in simplifying drug supply management (procurement, storage and distribution), doctor's prescription and patient consumption of anti-TB medications, this systematic review provides evidence that FDC formulations are not superior to separate-drug formulations for preventing treatment failure or disease relapse. Furthermore, there is no evidence that FDC formulations will improve patient compliance, and inconsistent evidence that FDC regimens improve treatment satisfaction. These findings may not be generalisable to settings with unstandardised or uncontrolled medical practice.

This systematic review of current evidence does not support the use of FDC formulations for the purpose of improving treatment outcomes among patients with active TB. To provide high-quality evidence for health policies and clinical decisions, further research on clinical effectiveness of FDC anti-TB formulations should utilise pragmatic trial designs to simulate real-world clinical practice while minimising confounding.

\section{Acknowledgements}

The authors thank Madhukar Pai (Dept of Epidemiology and Biostatistics, McGill University, Montreal, Canada) and Andrea Benedetti (Montreal Chest Institute, McGill University) for assistance in data analysis; Ioana Nicolau and Daniel Magin (McGill University), Idun Liu (Montreal Chest Institute) and Antje Bier (Royal Victoria Hospital, McGill University) for support in translation of foreign language articles; and Christian Lienhardt (Stop TB Partnership, World Health Organization, Geneva, Switzerland) and Otto Braendli (Swiss Lung Foundation, Wald, Switzerland) for providing additional information related to their studies.

\section{References}

World Health Organization (WHO). Global tuberculosis control: WHO report 2012. WHO/HTM/TB/2012.6. World Health Organization (WHO). Multidrug and extensively drug-resistant TB (M/XDR-TB): 2010 global report on surveillance and response. WHO/HTM/TB/2010.3.

3 Mitchison DA. How drug resistance emerges as a result of poor compliance during short course chemotherapy for tuberculosis. Int J Tuberc Lung Dis 1998; 2: 10-15. 
van Altena R, van Soolingen D, van der Werf TS. Isoniazid resistant TB and non-compliance. Thorax 2004; 59 : 1098. Norval PY, Blomberg B, Kitler ME, et al. Estimate of the global market for rifampicin-containing fixed-dose combination tablets. Int J Tuberc Lung Dis 1999; 3: Suppl. 3, S292-S300.

6 Bangalore S, Kamalakkannan G, Parkar S, et al. Fixed-dose combinations improve medication compliance: a metaanalysis. Am J Med 2007; 120: 713-719.

7 The promise and reality of fixed dose combinations with rifampicin. A joint statement of the International Union Against Tuberculosis and Lung Disease and the Tuberculosis Programme of the World Health Organization. Tuber Lung Dis 1994; 75: 180-181.

8 Shishoo CJ, Shah SA, Rathod IS, et al. Impaired bioavailability of rifampicin in presence of isoniazid from fixed dose combination (FDC) formulation. Int J Pharm 2001; 228: 53-67.

9 Pillai G, Fourie PB, Padayatchi N, et al. Recent bioequivalence studies on fixed-dose combination anti-tuberculosis drug formulations available on the global market. Int J Tuberc Lung Dis 1999; 3: Suppl. 3, S309-S316.

10 Milán-Segovia RC, Domínguez-Ramírez AM, Jung-Cook H, et al. Relative bioavailability of rifampicin in a threedrug fixed-dose combination formulation. Int J Tuberc Lung Dis 2010; 14: 1454-1460.

11 Singh S, Mariappan TT, Shankar R, et al. A critical review of the probable reasons for the poor variable bioavailability of rifampicin from anti-tubercular fixed-dose combination (FDC) products, and the likely solutions to the problem. Int J Pharm 2001; 228: 5-17.

12 Assuring bioavailability of fixed-dose combinations of anti-tuberculosis medications. A joint statement of the International Union Against Tuberculosis and Lung Disease and the World Health Organization. Int J Tuberc Lung Dis 1999; 3: Suppl. 3, S282-S283.

13 WHO Model List of Essential Medicines. 17th list (March 2011). WHO Drug Information 2011.

14 Moulding TS, Le HQ, Rikleen D, et al. Preventing drug-resistant tuberculosis with a fixed dose combination of isoniazid and rifampin. Int J Tuberc Lung Dis 2004; 8: 743-748.

15 Suryanto AA, van den Broek J, Hatta M, et al. Is there an increased risk of TB relapse in patients treated with fixeddose combination drugs in Indonesia? Int J Tuberc Lung Dis 2008; 12: 174-179.

16 Lienhardt C, Cook SV, Burgos M, et al. Efficacy and safety of a 4-drug fixed-dose combination regimen compared with separate drugs for treatment of pulmonary tuberculosis: the Study C randomized controlled trial. JAMA 2011; 305: 1415-1423.

17 Bartacek A, Schütt D, Panosch B, et al. Comparison of a four-drug fixed-dose combination regimen with a single tablet regimen in smear-positive pulmonary tuberculosis. Int J Tuberc Lung Dis 2009; 13: 760-766.

18 World Health Organization (WHO). Treatment of tuberculosis: guidelines. 4th Edn. (WHO/HTM/TB/2009.420). Geneva, World Health Organization, 2009.

19 International Standards for Tuberculosis Care (ISTC). 2nd Edn. The Hague, Tuberculosis Coalition for Technical Assistance, 2009.

20 American Thoracic Society, CDC, Infectious Diseases Society of America. Treatment of tuberculosis. MMWR Recomm Rep 2003; 52: 1-77.

21 Higgins JPT, Green S, eds. Cochrane Handbook for Systematic Reviews of Interventions. Chichester, The Cochrane Collaboration, 2011.

22 Research Committee of the Tuberculosis Association of India. Fifth tuberculosis association of India: short course chemotherapy trial. Ind J Tuberc 1989; 36: 95-101.

23 Cowie RL, Brink BA. Short-course chemotherapy for pulmonary tuberculosis with a rifampicin-isoniazidpyrazinamide combination tablet. S Afr Med J 1990; 77: 390-391.

24 Controlled trial of 2, 4, and 6 months of pyrazinamide in 6-month, three-times-weekly regimens for smear-positive pulmonary tuberculosis, including an assessment of a combined preparation of isoniazid, rifampin, and pyrazinamide. Results at 30 months. Hong Kong Chest Service/British Medical Research Council. Am Rev Respir Dis 1991; 143: 700-706.

25 Acceptability, compliance, and adverse reactions when isoniazid, rifampin, and pyrazinamide are given as a combined formulation or separately during three-times-weekly antituberculosis chemotherapy. Hong Kong Chest Service/British Medical Research Council. Am Rev Respir Dis 1989; 140: 1618-1622.

26 Glatthaar E, Summers FS, Carlier ND. A comparative community-based therapy trial with a single combination product (Rifater-80). J Compr Health 1991; 2: 155-158.

27 Macnab MF, Bohmer PD, Seager JR. Evaluation of the 3-drug combination, Rifater, versus 4-drug therapy in the ambulatory treatment of tuberculosis in Cape Town. S Afr Med J 1994; 84: 325-328.

28 Chaulet P, Boulahbal F. Essai clinique d'une combinaison en proportions fixes de trios medicaments dans le traitement de la tuberculose. [Clinical trial of a combination of three drugs in fixed proportions in the treatment of tuberculosis. Groupe de Travail sur la Chimiothérapie de la Tuberculose.] Tuber Lung Dis 1995; 76: 407-412.

29 Agounitestane D, Chiheb M, Khaled S, et al. Essai therapeutique d'une combinaison de trois medicaments essentiels dans la chimiotherapie courte de la tuberculose. Resultats six mois après la fin du traitement. [A therapeutic trial of a combination of 3 essential drugs in a short course of chemotherapy in tuberculosis. Results 6 months after the end of treatment.] Rev Mal Respir 1990; 7: 209-213.

30 Bellabas M, Khaled S, Khaled NA, et al. Essai therapeutique d'une combinaison d'isoniazide, de rifampicine et de pyrazinamide au cours des deux premiers mois du traitement de la tuberculose pulmonaire. [Therapeutic trial of a combination of isoniazid, rifampicin and pyrazinamide in the first 2 months of treatment of pulmonary tuberculosis.] Rev Mal Respir 1989; 6: 59-64.

31 Zhang LX, Kan GQ, Tu DH, et al. Fixed-dose combination chemotherapy versus multiple, single-drug chemotherapy for tuberculosis. Curr Ther Res 1996; 57: 849-856.

32 Zhu L, Yan B, Ma W. [Controlled clinical study on efficacy of fixed-dose compounds rifater/rifinah in antituberculous chemotherapy.] Zhonghua Jie He He Hu Xi Za Zhi 1998; 21: 645-647.

33 Teo SK. Assessment of a combined preparation of isoniazid, rifampicin and pyrazinamide (Rifater) in the initial phase of chemotherapy in three 6-month regimens for smear-positive pulmonary tuberculosis: a five-year followup report. Int J Tuberc Lung Dis 1999; 3: 126-132.

34 Assessment of a daily combined preparation of isoniazid, rifampin, and pyrazinamide in a controlled trial of three 6-month regimens for smear-positive pulmonary tuberculosis. Singapore Tuberculosis Service/British Medical Research Council. Am Rev Respir Dis 1991; 143: 707-712. 
Su WJ, Perng RP. Fixed-dose combination chemotherapy (Rifater/Rifinah) for active pulmonary tuberculosis in Taiwan: a two-year follow-up. Int J Tuberc Lung Dis 2002; 6: 1029-1032.

36 Munteanu I, Husar I, Didilescu C, et al. Consideratii privind eficacitatea regimurilor terapeutice ce au in componentă combinatii fixe Rifampicină-Izoniazidă in tratamentul tubeculozei pulmonare. [Considerations about the efficiency of treatment regimens with fixed Rifampicin-Isoniazid combinations in pulmonary tuberculosis.] Pneumologia 2004; 53: 23-25.

$37 \mathrm{Xu} \mathrm{W}-\mathrm{G}, \mathrm{Lu} \mathrm{W}, \mathrm{He} \mathrm{H}-\mathrm{J}$, et al. [Randomized control study on domestic fixed-dose combinations in the initial treatment of smears positive tuberculosis.] Zhonghua Jie He He Hu Xi Za Zhi 2004; 27: 690-693.

38 Gravendeel JM, Asapa AS, Becx-Bleumink M, et al. Preliminary results of an operational field study to compare side-effects, complaints and treatment results of a single-drug short-course regimen with a four-drug fixed-dose combination (4FDC) regimen in South Sulawesi, Republic of Indonesia. Tuberculosis 2003; 83: 183-186.

39 Sokolova GB, Semenova OV, Bogadel'nikova IV, et al. Mairin-P v kompleksnoi terapii tuberkuleza. [Myrin P in the combined treatment of tuberculosis.] Antibiot Khimioter 2002; 47: 22-27.

40 Brändli O, Dreher D, Morger D. Ergebnisse der Tuberkulose-Kurzzeittherapie mit Isoniazid, Rifamipicin und Pyrazinamid. [Results of short-term tuberculosis therapy with isoniazid, rifampicin and pyrazinamide.] Schweiz Med Wochenschr 1993; 123: 1300-1306.

41 Brändli O, Haegi V, Villiger B, et al. Kurzzeittherapie der Lungentuberkulose mit einer fixen Kombination von Isoniazid, Rifampicin und Pyrazinamid. Verlaufsbeobachtung über 2 Jahre. [Short-term therapy of lung tuberculosis using a fixed combination of isoniazid, rifampicin and pyrazinamide. Results after 2 years.] Schweiz Med Wochenschr 1989; 119: 299-305.

42 Punnotok J, Pumprueg U, Chakorn TA. A comparison of two short course tuberculosis chemotherapy regimens, both using Rifater during an intensive phase, with a 3 year follow-up. J Med Assoc Thai 1995; 78: $298-304$.

43 Churchyard GJ, Corbett EL, Kleinschmidt I, et al. Drug-resistant tuberculosis in South African gold miners: incidence and associated factors. Int J Tuberc Lung Dis 2000; 4: 433-440.

44 Haynes RB, Ackloo E, Sahota N, et al. Interventions for enhancing medication adherence. Cochrane Database Syst Rev 2008; 2: CD000011.

45 Chalkidou K, Tunis S, Whicher D, et al. The role for pragmatic randomized controlled trials (pRCTs) in comparative effectiveness research. Clin Trials 2012; 9: 436-446.

46 Tunis SR, Stryer DB, Clancy CM. Practical clinical trials: increasing the value of clinical research for decision making in clinical and health policy. JAMA 2003; 290: 1624-1632.

47 Moher D, Liberati A, Tetzlaff J, et al. Preferred reporting items for systematic reviews and meta-analyses: the PRISMA statement. PLoS Med 2009; 6: e1000097. 